\title{
The Effect of Biodegradable Scaffolds for Osteochondral Repair on the Immobilized Rabbit Knee
}

\author{
Chih-Chan Lin ${ }^{1,2}$, Nai-Jen Chang ${ }^{3}$, Ming-Long Yeh ${ }^{4,5}$, Chien-Feng Li ${ }^{6}$ and Yung-Chang Lin ${ }^{2 *}$ \\ ${ }^{1}$ Laboratory Animal Center, Department of Medical Research, Chi-Mei Medical Center, 901 Zhonghua, Yongkang Dist., Tainan City 701, Taiwan \\ ${ }^{2}$ Department of Veterinary Medicine, National Chung Hsing University, 250 KuoKuang Rd., South Dist., Taichung City 402, Taiwan \\ ${ }^{3}$ Department of Sports Medicine, Kaohsiung Medical University, 100, Shih-Chuan 1st Rd., Kaohsiung City, Taiwan \\ ${ }^{4}$ Department of Biomedical Engineering, National Cheng Kung University, 1 University Rd., Tainan City 701, Taiwan \\ ${ }^{5}$ Medical Device Innovation Center, National Cheng Kung University, Tainan City 701, Taiwan \\ ${ }^{6}$ Division of Clinical Pathology, Department of Pathology, Chi-Mei Medical Center, 901 Zhonghua Rd., Yongkang Dist., Tainan City 701, Taiwan
}

\begin{abstract}
Background: An initial constrained weight-bearing period achieved via immobilization $(\mathrm{Imm})$ is recommended during osteochondral regeneration to avoid tissue re-injury. However, remedying osteochondral defects with a scaffoldbased treatment and Imm intervention has yet to be studied in osteochondral defect models.

Therefore, we investigated the combined outcome of short-term Imm on osteochondral defects with and without porous poly (lactic-co-glycolic acid) (PLGA) scaffold implants.

Methods: Twenty-four rabbits were divided into empty defect (ED) and PLGA-implanted (PI) groups. Each rabbit's unilateral knee, which contained an osteochondral defect, was immobilized at full extension with a splint for 2 weeks after surgery and subsequently remobilized until sacrifice at 4 weeks or 12 weeks post surgery. The health status; gross appearance; histological analysis detailing collagen, glycosaminoglycan, and matrix metalloproteinase (MMP)13 expression levels; and micro-CT images of the rabbits were evaluated.

Results: At 4 weeks, the ED and PI groups both exhibited degenerative cartilage features, including an irregular joint surface, fibrous or fibrocartilage formation, decreased glycosaminoglycan (GAG) expression, and increased MMP-13 expression. However, at 12 weeks, the PI group exhibited significantly higher GAG expression, lower MMP13 expression, and more bony deposition matrix and trabecular bone than the ED group.
\end{abstract}

Conclusion: Our findings indicate that the short-term Imm is harmful to articular cartilage; however, PLGA grafts could provide a matrix for tissue regeneration.

Keywords: Cartilage; Immobilization; Scaffold; Knee; Tissue engineering; Rabbit

\section{Introduction}

Osteochondritis dissecans involving the cartilage and bone layers, which results in trauma, joint instability, and insufficient blood flow to the subchondral area, is initiated by osteocartilaginous separation and leads to osteoarthritis (OA), impairment and disability [1]. In addition, once injured, the vascular articular cartilage has poor regenerative capacity compared with other connective tissues. Thus, defects often progress into OA. As a solution, clinicians have regularly used corrective therapies, 13 including autologous chondrocyte implantation or osteochondral graft substitutes [2,3]. Hyaline-like regenerative tissue has been proposed as an essential marker for the long-term clinical success of these cartilage repair methods. However, challenges remain, including poor integration of grafts with the host cartilage and formation of fibro cartilage [4]. Alternatively, some studies have shown promising regenerative outcomes by using biocompatible and biodegradable scaffold-based strategies $[5,6]$. Researchers have hypothesized that the functional porous scaffolds provide a shortterm structure and space that enhances tissue regeneration, increases Extracellular Matrix (ECM) synthesis and remodelling, and withstands external loading during tissue repair. A degradable poly (lacticco-glycolic acid) (PLGA) biomaterial exhibits the aforementioned properties and has been extensively adopted in several pre-clinical applications, including osteochondral engineering [5,7]. Traditional reconstruction and rehabilitation procedures recommend that patients progress from constrained weight bearing (CWB) to full weight bearing (FWB). CWB of the repaired tissue is critical to decrease pain and movement in the defect region initially and can be achieved by using a cast, brace or splint for short-term immobilization (Imm) [8]. For most defects, Imm is generally applied for the first 2 to 4 weeks after surgery [9]. Imm is recommended during the treatment of injuries to protect healing tissues from unexpected destructive forces and prevent complications such as reconstruction failure. Imm during the CWB phase has been reported to cause deleterious effects, including synovial adhesions, articular cartilage erosion, thinner cartilage, depressed total glycosaminoglycan (GAG) and disuse muscle atrophy $[6,10-13]$. However, some published studies have shown no difference in macroscopic exams of cartilage quantity and soft tissue atrophy prevention between CWB and FWB groups [14-16]. Overall, repair outcomes have not improved with Imm treatment $[8,10,12]$. However, remedying osteochondral defects with a scaffold-based treatment and Imm has yet to be studied in osteochondral defect animal models. Therefore, we aimed to examine the combined outcome of Imm and a PLGA scaffold on osteochondral regeneration creating by subchondral

*Corresponding author: Yung-Chang Lin, Department of Veterinary Medicine, National Chung Hsing University, No.250, KuoKuang Rd., South Dist., Taichung City 402, Taiwan, E-mail: ylin@nchu.edu.tw, harry-2001@yahoo.com.tw

Received December 07, 2013; Accepted December 30, 2013; Published January 07, 2014

Citation: Lin CC, Chang NJ, Yeh ML, Li CF, Lin YC (2014) The Effect of Biodegradable Scaffolds for Osteochondral Repair on the Immobilized Rabbit Knee. Surgery Curr Res 4: 158. doi:10.4172/2161-1076.1000158

Copyright: (c) 2014 Lin CC, et al. This is an open-access article distributed under the terms of the Creative Commons Attribution License, which permits unrestricted use, distribution, and reproduction in any medium, provided the original author and source are credited. 
drilling in rabbits. Scaffold-based treatment and Imm has yet to be studied in osteochondral defect animal models. Therefore, we aimed to examine the combined outcome of Imm and a PLGA scaffold on osteochondral regeneration creating by subchondral drilling in rabbits.

\section{Materials and Methods}

\section{Animal procedures}

Twenty-four 4-to 5-month-old New Zealand White male rabbits weighing 2-3 $\mathrm{kg}$ were used in this study. The sample size was six rabbits for each group at each time point. All surgical procedures were permitted under the Animal Care and Use Committees of the National Cheng Kung University in Taiwan. First, the rabbits were anesthetized with a subcutaneous injection of Zoletil 50 (25 mg/kg) (Virbac, France), followed by intubation and maintenance with a mixture of oxygen/ nitrous oxide $(1 / 0.413 \mathrm{~L} / \mathrm{min})$ and $2 \%$ isoflurane (Panion \& BF Biotech Inc., Taiwan). Under anesthesia, both knees were shaved, disinfected and covered with a sterilized drape. The joint capsule was opened through an anteromedial knee incision. The femoral trochlear groove, which was a lower weight-bearing (LWB) region, was exposed after laterally dislocating the patella. A full-thickness osteochondral defect was created on the femoral trochlear groove by drilling a 3-mm-diameter hole that was $3 \mathrm{~mm}$ deep. Concurrently, during the drilling procedure, the defect was irrigated to prevent from thermal damage. Next, the joint space was cooled and washed using saline irrigation again, and debris was removed from the defect region. Then, the rabbits were randomly divided into the Empty Defect (ED) and PLGA-implanted (PI) groups with or without Imm treatment, which was applied for only one leg of a rabbit. The $3 \mathrm{D}$ porous PLGA grafts (PLA/PGA: $85 / 15$, porosity: $>90 \%$, pore size: $300-500 \mu \mathrm{m}$ (Figure 1a), and wet compressive modulus: 0.65 $\mathrm{MPa}$ ) were fabricated using the salt-leaching and lyophilized methods described in the previous studies of Chang et al. [5]. In the PI group, a PLGA graft that was pre-sterilized in $75 \%$ ethanol, followed by sterilized PBS wash several times, was implanted into the defect hole by press-fit fixation (Figure 1b) and repositioned in the patellar position. The joint capsule and subcutaneous tissues were closed using 3-0 absorbable Vicryl sutures and 3-0 nylon sutures, respectively. Then, the knee was fixed using a splint positioned at the knee extension and ankle plantarflexion for 2 consecutive weeks (Figure 1c), followed by free cage activity. The Imm status of the rabbits was monitored by a clinical staff member (NJ Chang) and a veterinarian (CC Lin) for the entire Imm treatment course. Following the surgery routinely used analgesic, antibiotic and anti-infection medicines -ketoprofen, enrofloxacin, and povidone-iodine, respectively --were administered for several days. All of the rabbits were observed with regard to appetite, wound healing, and proper functional activity after surgery. The rabbits were euthanized 4 or 12 weeks after surgery, and their gross appearance, histology and $\mu \mathrm{CT}$ images were evaluated.

\section{Gross appearance and histological assessments}

Either 4 or 12 weeks after surgery, knee joint capsules were opened carefully to avoid damage to the adjacent cartilage. Then, the gross appearance was blindly assessed by two medical staff members (N) Chang and CC Li) using the modified Wayne's grading scale [16]. The three main scoring parameters for gross appearance included coverage (>75\% fill, 4 points (pt); 50-75\% fill, 3 pt; $25-50 \%$ fill, 2 pt; $<25 \%$ fill, 1 pt; $0 \%$ fill, 0 pt), tissue colour (normal/whitish, 4 pt; $25 \%$ yellow/brown/ reddish, $3 \mathrm{pt}$; $50 \%$ yellow/brown/reddish, $2 \mathrm{pt}$; $75 \%$ yellow/brown/

(d)

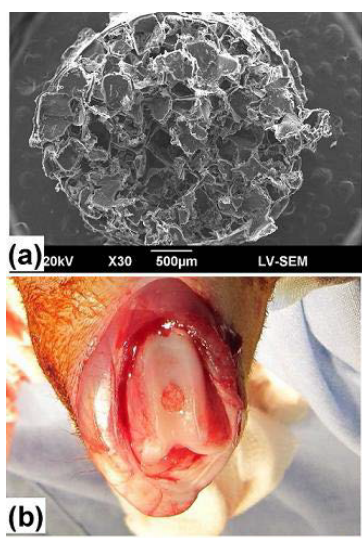

ED

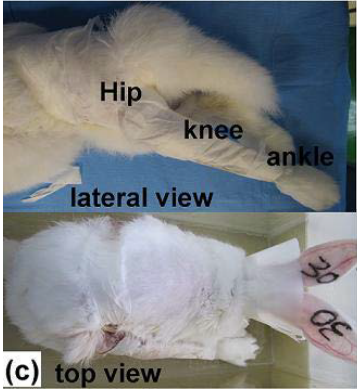

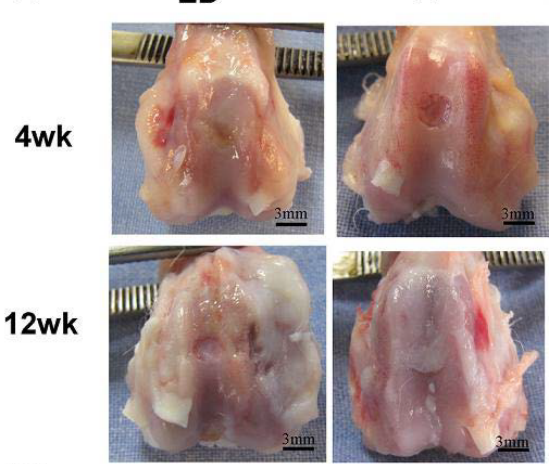

12wk

(e)

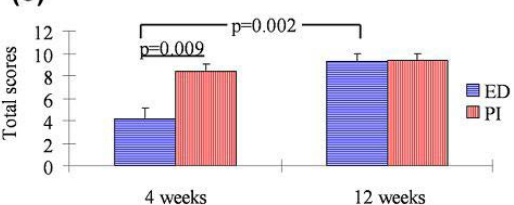

Figure 1: (a) Scanning electron microscopy image showing the gross appearance of a 3D porous PLGA graft, (b) Pre-sterilized PLGA graft implanted into a 3-mmdiameter defect hole by press-fit fixation

(c) A rabbit's knee restricted by a splint positioned at the knee extension for 2 weeks post surgery.

(d) Images taken 4 and 12 weeks after surgery showing the gross appearance of the healing osteochondral defect for the ED and PI groups.

(e) The quantitative scores of gross appearance calculated 4 and 12 weeks after surgery. 
reddish, $1 \mathrm{pt} ; 100 \%$ yellow/brown/reddish, $0 \mathrm{pt}$ ) and surface smoothness (smooth/level with normal tissue, $4 \mathrm{pt}$; smooth/raised compared with normal tissue, $3 \mathrm{pt}$; irregular $25-50 \%, 2 \mathrm{pt}$; irregular $50-75 \%, 1 \mathrm{pt}$; irregular $>75 \%, 0 \mathrm{pt}$ ). The maximum possible score was $12 \mathrm{pt} .2 .3$ Histological and Immunohistochemical Processing After harvesting, the histological sections were processed by the Department of Pathology at the Chi-Mei Medical Center. The samples underwent formalin fixation, dehydration, decalcification, and infiltration and paraffin embedding. The samples $(4 \mu \mathrm{m}$ thick) were stained with hematoxylin and eosin (H\&E) to evaluate inflammatory cells, Masson's trichrome to assess total collagen content and alignment, and Alcian blue to identify the GAG content [17]. The sections were examined under light microscopy (Olympus BX51, Japan) and imaged using a digital CCD camera (Olympus DP70, Japan). In addition, immunohistochemistry (IHC) was used to measure matrix metalloproteinase (MMP)-13 expressions at postsurgical week 4 and 12. MMP-13 can indicate mitochondrial dysfunction and degenerative cartilage in a repaired area [18]. All of the processes were followed the manufacturer's protocol (BioSB, USA). All sections were deparaffinised, gradient rehydrated and incubated with the enzymatic digest Proteinase K for $60 \mathrm{~min}$ [17]. To avoid endogenous peroxidise over activation, all sections were treated with the peroxidise-blocking reagent included in the Rabbit/Mouse HRPDAB detection system (catalogue number BSB0205, BioSB, USA) at room temperature for $10 \mathrm{~min}$. Subsequently, the samples were treated using an anti-MMP-13 antibody (dilution factor 1:100; Bio world Technology, Inc., USA) at room temperature overnight in a humidified chamber. The Rabbit/Mouse HRP-DAB Polymer detection system was used as the secondary antibody at room temperature for $30 \mathrm{~min}$. After washing with $\mathrm{PBS}$, the signal was identified as a brown precipitate using DAB (3, 3'diaminobenzidine) substrate (BioSB, USA). The samples were counterstained with haematoxylin (BioSB, USA), dehydrated and mounted.

\section{Bony matrix measurements using $\mu \mathrm{CT}$}

The osteochondral samples were placed on a sample holder with the femur axis perpendicular to the scanning plane. Then, the femoral trochlear grooves were scanned using in vivo non-destructive $\mu \mathrm{CT}$ (Sky scan 1076 scanner, Kontich, Belgium) to acquire qualitative images and quantitatively assess the properties of the new bony matrix including bone volume per tissue volume (BV/TV) and trabecular thickness (Tb. $\mathrm{Th}$ ). The voltage and beam current of the X-ray source were $50 \mathrm{kV}$ and $160 \mu \mathrm{A}$, respectively. The samples were scanned through a $360^{\circ}$ rotation angle with a rotation interval of $1^{\circ}$ at $18-\mu \mathrm{m}$ resolution [17]. Sky can software was used to reconstruct the image data and analyze a 3-mmdiameter region of interest (ROI) within the repaired site.

\section{Statistics}

All of the data are shown as the mean \pm standard error of the mean (SEM). Statistical analyses were performed using the SPSS v17.0 Windows software package. Normal distribution of data was confirmed by the Shapiro-Wilk test before ran the statistics. To analyze the gross appearance scores, BV/TV and Tb.Th, between the ED and PI groups or the two time points (postoperative weeks 4 and 12), a two-tailed unpaired Student's $t$ test was performed. A significant difference was set at $\mathrm{p}<0.05$.

\section{Results}

\section{Rabbit health condition after surgery}

Due to obstructed gastrointestinal tract, 2 of the 24 rabbits were excluded from the study. Both two rabbits belonged to the ED group. The average recovery time to fully functional activity with normal behaviour, eating and drinking, was approximately 2-3 weeks. Additionally, rabbits usually could act during unilateral knee immobilized. Acute inflammation responses and a lack of surgical incision wound closure occurred in all rabbits after removing the splint 2 weeks after surgery (data not shown). Mild to moderate unhealed surgical incision wounds were still present 4 weeks after surgery, but complete surgical incision wound closure was seen after 12 weeks. Physical examinations revealed a limited range of motion, joint stiffness and palpated muscle atrophy 4 weeks after surgery, but all rabbits had regained their pre-operative health status 12 weeks after the operation.

\section{Gross appearance}

In general, degeneration of the joint, an irregular cartilage surface, synovitis, and soft or hard osteophytes were observed (Supplementary Figure 1). At week 4 post surgery the defects in both the ED and PI groups were filled with reddish or yellow-brown granular reparative tissues at the margins, with a distinguishable hollow at the center of
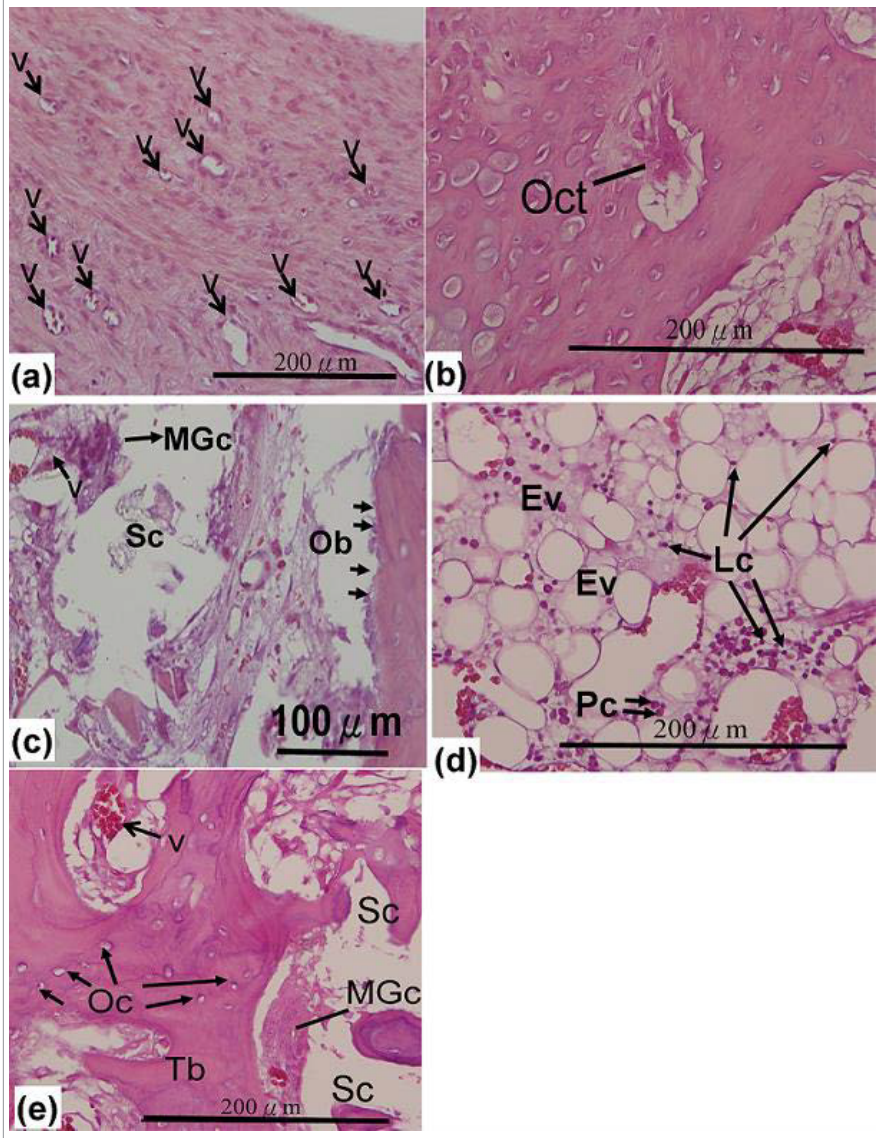

Figure 2: At week 4, the ED group displayed

(a) Vascular infiltration ( $v$ ) in the upper fibrous tissues (neo-cartilage layer) (b) Osteoclast (Oct) in the bone region.

(c) At week 4, the PI group displayed delineated osteoblasts $(\mathrm{Ob})$ surrounding the bony matrix and multinucleated giant cells (MGc) around the scaffold (Sc).

(d) At week 12, the ED group showed an inflammatory response including edema vesicles (Ev), plasma cells (Pc) and lymphocytes (Lc).

(e) At week 12, the PI group displayed a higher number of osteocytes (Oc) associated with mature trabecular bone $(\mathrm{Tb})$ and vascular formation $(\mathrm{v})$ inside the scaffold (Sc) while the rest of the PLGA scaffold was still undergoing endocytosis by MGc. 
the defect. However, the PI group displayed more newly formed tissues around the defect and less severe adjacent cartilage erosion than the ED group (Figure 1d). At week 12, the ED group defects were almost filled in with newly formed fibrous tissue, but the edges of the defects were still visible (Figure 1d). Moreover, a severe hard osteophyte had formed on the patellar groove. In contrast, the defects of the PI group were completely filled in with raised, opaque-white tissue that extended into adjacent cartilage (Figure 1d). At week 4, the total scores for the ED group ( $4.2 \pm 1.0)$ were significantly lower than the PI group scores $(8.4 \pm 9.3)(p=0.009)$. However, the total scores were not significantly different at week 12 . In addition, the ED group exhibited significantly higher total scores at week $12(9.3 \pm 0.6)$ than at week $4(\mathrm{p}=0.002)$, but this difference was not seen in the PI group.

\section{Histological findings}

In the ED group at week 4, inflammatory cells, including plasma cells and lymphocytes were observed in overlying fibrous tissues, vascular infiltration was observed in the upper fibrous tissues (Figure 2a), and osteoclasts were found in the bone region (Figure 2b). In addition, regenerated tissue was dominated by irregular and disorganized fibrous tissue and was depressed relative to the adjacent cartilage. Furthermore, fibroblast-like cells were observed (Figure 3). Reduced GAG expression was seen in the repaired region and adjacent cartilage (Figure 3 ), and degenerative proteinase MMP-13 expression was observed in the repaired tissues (Figure 4a). In contrast, osteoblasts surrounding the bony matrix and multinucleated giant cells (MGc) around the scaffold (Sc) were observed in the PI group (Figure 2c). In addition, newly formed tissues had filled in the pores of the construct and cracks, GAG syntheses (Figure 3) and MMP-13 were observed (Figure 4b), and the boundary between the repair site and the surrounding tissue was visible (Figure 3). At postoperative week 12, the inflammatory response, characterized by edematous vesicles, plasma cells and lymphocytes was still visible in the ED group (Figure 2d). The ED group had fewer fibrillated tissues, thinner and more disorganized total collagen, and less GAG content in the repaired region. In addition, the adjacent articular cartilage became thinner, lost GAG content (Figure 3) and expressed MMP-13 (Figure 4c) on repaired cartilage-layer tissues. Moreover, a disordered osseous matrix consisting mainly of compact bone was observed in the regenerated tissue (Figure 5a). In contrast, the PI group displayed a higher number of osteocytes associated with mature trabecular bone inside the remaining porous PLGA scaffold (Figure 2e). The PI group exhibited slight fissures in the cellular region of the articular surface mixed with "scaling" fibrous tissues and fibro cartilage structures (Figure 3). However, the GAG content appeared to be hyper-regulated in both the regenerated and adjacent cartilage (Figure 3). MMP-13 was slightly expressed in fibrous tissues, but not expressed in the 17 underlying fibro cartilage tissues or surrounding articular cartilage (Figures $4 \mathrm{~d}$ and $4 \mathrm{e}$ ). Additionally, the neo-formed tissues were better integrated into the adjacent cartilage and bone (Figures 3 and 5a).

\section{$\mu \mathrm{CT}$ analysis}

At week 4, the ED and PI groups had both generated newly mineralized bone from the edge of the defect (Figure 5a) and exhibited an osteoporosis-like appearance in the host bone regions. Significant differences in BV/TV and $\mathrm{Tb}$. Th values were not found between the two groups (Figures $5 \mathrm{~b}$ and $5 \mathrm{c}$ ), both $\mathrm{p}>0.05$ ) at week 12 , an improved osseous matrix with trabecular bone was recognized in the PI group, while the ED group displayed mostly compact bone (Figure 5a). The PI group also regained calcium deposition in the adjacent bone area. The BV/TV in the PI group (43.5 \pm 5.1$)$ was significantly higher than the ED group $(23.8 \pm 2.1)(\mathrm{p}=0.005)$ (Figure $5 \mathrm{~b})$. Moreover, both the PI group $(\mathrm{p}=0.003)$ and ED group $(\mathrm{p}=0.043)$ at week 12 had significantly higher BV/TV than at week 4 . At week 12, the PI group exhibited a significantly lower $\mathrm{Tb}$. Th value than the ED group $(\mathrm{p}=0.007)$. The PI group $(\mathrm{p}=0.002)$ and ED group $(\mathrm{p}=0.001)$ had significantly higher $\mathrm{Tb}$. Th values in week 12 than week 4 (Figure $5 \mathrm{c}$ ).

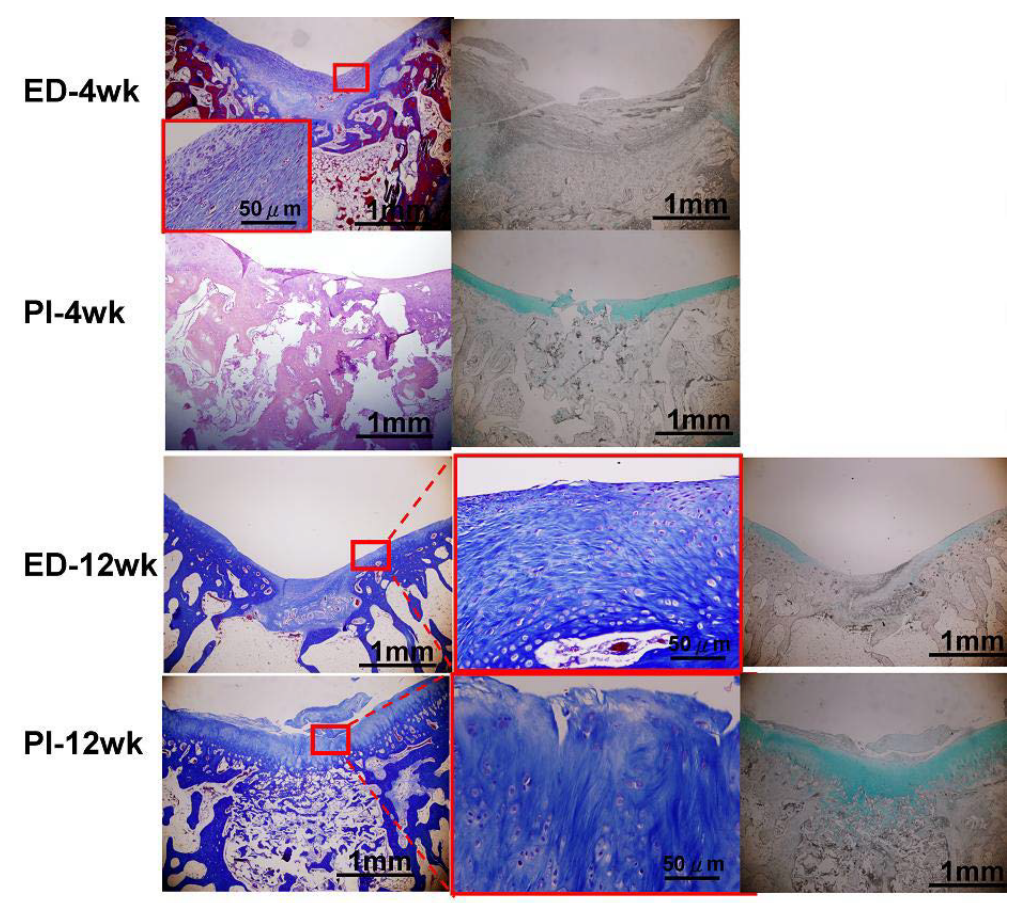

Figure 3: Histological images following H\&E, Masson's trichrome (dark blue colour), and Alcian blue (light green) staining. The 1 red squares indicate regional magnification. 


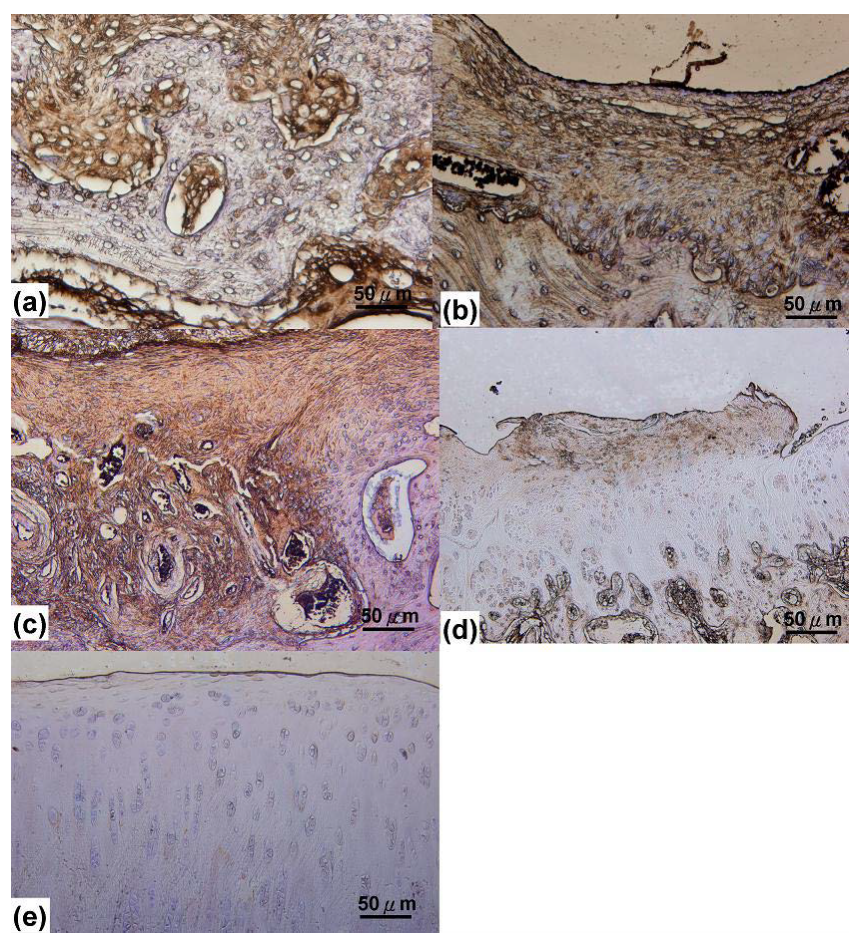

Figure 4: At week 4, positive MMP-13 expression (brown colour) was observed in the upper repaired tissues of the

(a) ED

(b) PI groups. At week 12, MMP-13 expression was higher in the

(c) ED group than the (d) PI group and (e) no MMP-13 expression was observed in the adjacent normal cartilage layer

\section{Discussion}

In clinical orthopaedics and rehabilitation, joint immobilization or CWB activities are common treatments during the initial healing phase to stabilize the repaired region and improve healing. However, Imm can also lead to unfavourable outcomes, including articular cartilage degeneration or soft tissue atrophy [19]. Previous studies have evaluated repairs following the use of various immobilization methods, but the processes that take place in the joint after immobilization have not been elucidated. Cartilage degeneration due to immobilization has been reported in LWB, weight bearing, and both LWB and weight bearing regions [20-22]. In this study, we exposed a defect in the femoral trochlear groove, where patellofemoral pain syndrome often occurs and results in OA, to LWB to evaluate the differences in the articular cartilage after immobilization and the effect of applying a scaffold graft into the defect. Additionally, because animals cannot be controlled with verbal cues telling them to not apply any weight to the affected limb, we adopted an alternate method of Imm. At postoperative week 4 in both the ED and PI groups, the rabbit Imm models showed severe, harmful changes in the articular cartilage and subchondral bone, including the presence of inflammatory cells, loss of GAG content in both the repaired and adjacent cartilage regions, strong expression of MMP-13, and osteoporosis-like characteristics that might have been caused by the absorption of bone from highly activate osteoclasts (Figures 2-5). Interestingly, at week 12, compared with the ED group, the PI group had more GAG content and relatively lower expression of MMP-13 --a specific proteinase that indicates OA in the osteochondral region in the regenerative tissues adjacent to the original cartilage region (Figures 2e-5) [18]. However, the overall cartilage surface in the PI group showed degenerative progress. The PLGA scaffolding may have relieved the progress of osteochondral degradation caused by the initial immobilization. We speculate that two mechanisms could be responsible for the results of this study. First, the various changes in the articular cartilage may have been due to the immobilization method. Immobilization methods are commonly classified into rigid fixation, which uses an internal or external fixator, or non-rigid fixation, which uses plaster or a splint $[23,24]$. Rigid fixation methods have been reported to cause more severe degenerative changes than non-rigid fixation methods, which allow for mechanical stimulation to the articular cartilage [23]. In a previous study, a rabbit's knee was immobilized through the tibia and femur at approximately $150-160^{\circ}$ flexions, which is different than the knee extension immobilization model used in this study. In our pilot study, the gross appearance of Imm knees showed that knee extension Imm was better than knee flexion Imm [6]. Knee extension Imm was selected for this study because this method mimicked clinical usage, increased blood circulation, prevented musculoskeletal necrosis and applied less compressive force on the regenerative tissues by the patella, which prevented progressive circulating damage. Second, the strong porous scaffold provided a matrix for nearby mesenchymal cell migration and proliferation and tissue regeneration. Cells from surrounding mesenchymal or subchondral bone marrow could migrate into the interconnected pores provided by the PLPA scaffold. Additionally, the immobilized rabbits in our model were remobilized to allow for free activity after 2 weeks of Imm. Joint motion in all groups returned in 12 weeks. Joint adhesion and contracture could have been Prevented because of instant settlement of lysosomal enzymes [25]. When the scaffold-based structure and free activity were combined, the cells may have been stimulated toward chondrogenesis and osteogenesis by biomechanical cues from the remaining patella. The outcome is consistent with the results from histology and $\mu \mathrm{CT}$ images (Figures 2-5). The results indicate that MMP-13 expression is the most important marker for cartilage degradation. At week 4, abundant expression of MMP-13 in the regenerative tissues was detected in both the ED and PI

(a)

ED

4 wk

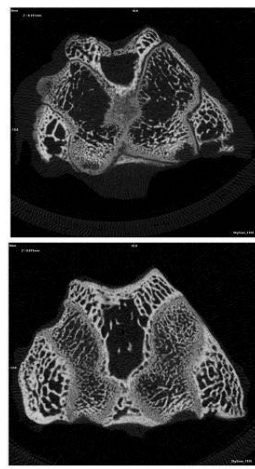

PI

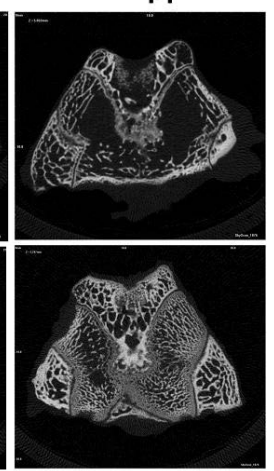

(b)

(c)
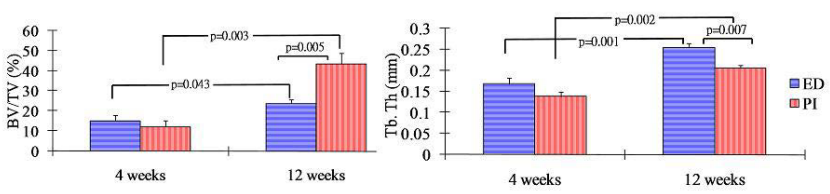

Figure 5: (a) The bone examination of 2D $\mu C T$ images

(b) The ratio of bone volume to tissue volume (BV/TV)

(c) The thickness of the trabecular bones (Tb. Th). 
groups (Figures $4 \mathrm{a}$ and $4 \mathrm{~b}$ ). At 12 weeks, persistent MMP- 13 secretion was remained in all tissues in the ED group (Figure 4c). In contrast, MMP-13 was only slightly expressed in the fibrous tissues overlying fibro cartilage (Figures 3 and $4 \mathrm{~d}$ ) and was barely expressed in adjacent cartilage (Figure 4e). Interestingly, MMP-13 expression appeared to be reduced after remobilization, but future examinations are needed to further evaluate this finding. In terms of bone regeneration, an improved osseous matrix with trabecular bone (i.e., BV/TV) was observed in the PI group compared with the ED group, which exhibited mostly compact bone (Figure 5). After 12 weeks, increased calcium deposition in the bone matrix was observed, and severe osteoporosis surrounding with the defect region was not observed in the PI group (Figure 5a). Thus, encouraging outcomes following postoperative activity were observed in this study. Some limitations existed in this study. First, the duration of Imm depends on the injury type, such as bone fracture or arthroplasty, and injury site, such as upper or lower extremities. Some previous studies applied 4-12 weeks of Imm on rabbits; however, we chose a relatively short period of 2 weeks of Imm to prevent severely damaging the surrounding tissues $[6,10]$. In this study, ROM reduction or contracture was not observed at 12 weeks, but a degenerative progress had occurred. Thus, the optimal Imm period should be addressed in future studies. Second, human patients undergoing Imm are encouraged to participate in activities such as isometric soft tissue contraction. However, rabbits cannot be directed to participate in such activities. Therefore, the healing process may be different in humans. Nevertheless, this study provides insight into the degradation caused by Imm treatment in rabbits and the benefit achieved by implanting an engineered graft. Third, long-term studies should be progressed and developed in the future. In our current study, the short-term imm is harmful to articular cartilage; however, PLGA grafts could provide a matrix for tissue regeneration. For future clinical scaffold-based osteochondral interventions, the Imm periods and early weight bearing activity should be concerned discreetlythe Imm periods and early weight bearing activity should be concerned discreetly.

\section{Acknowledgments}

This work was financially supported by the National Science Council of Taiwan (100-2627-B006-009)

\section{References}

1. Davin J, Selfe J (2006) Osteochondritis dessicans: a complex case of anterior knee pain. Man Ther 11: 157-161.

2. Pestka JM, Bode G, Salzmann G, Südkamp NP, Niemeyer P (2012) Clinical outcome of autologous chondrocyte implantation for failed microfracture treatment of full-thickness cartilage defects of the knee joint. Am J Sports Med 40: 325-331.

3. Könst YE, Benink RJ, Veldstra R, van der Krieke TJ, Helder MN, et al. (2012) Treatment of severe osteochondral defects of the knee by combined autologous bone grafting and autologous chondrocyte implantation using fibrin gel. Knee Surg Sports Traumatol Arthrosc 20: 2263-2269.

4. Martin I, Miot S, Barbero A, Jakob M, Wendt D (2007) Osteochondral tissue engineering. J Biomech 40: 750-765.

5. Chang NJ, Jhung YR, Issariyakul N, Yao CK, Yeh ML (2011) Synergistic Stimuli by Hydrodynamic Pressure and Hydrophilic Coating on PLGA Scaffolds for Extracellular Matrix Synthesis of Engineered Cartilage. J Biomater Sci Polym Ed.

6. Arakaki K, Kitamura N, Kurokawa T, Onodera S, Kanaya F, et al. (2011) Joint immobilization inhibits spontaneous hyaline cartilage regeneration induced by a novel double-network gel implantation. J Mater Sci Mater Med 22: 417-425.

7. Lü JM, Wang X, Marin-Muller C, Wang H, Lin PH, et al. (2009) Current advances in research and clinical applications of PLGA-based nanotechnology. Expert Rev Mol Diagn 9: 325-341.

8. Trudel G, Seki M, Uhthoff HK (2000) Synovial adhesions are more important than pannus proliferation in the pathogenesis of knee joint contracture after immobilization: an experimental investigation in the rat. J Rheumatol 27: 351 357.

9. Reinold MM, Wilk KE, Macrina LC, Dugas JR, Cain EL (2006) Current concepts in the rehabilitation following articular cartilage repair procedures in the knee. $J$ Orthop Sports Phys Ther 36: 774-794.

10. Maxwell LC, Enwemeka CS (1992) Immobilization-induced muscle atrophy is not reversed by lengthening the muscle. Anat Rec 234: 55-61.

11. Ando A, Hagiwara Y, Chimoto E, Hatori K, Onoda Y, et al. (2008) Intra-articular injection of hyaluronan diminishes loss of chondrocytes in a rat immobilizedknee model. Tohoku J Exp Med 215: 321-331.

12. Djurasovic M, Aldridge JW, Grumbles R, Rosenwasser MP, Howell D, et al. (1998) Knee joint immobilization decreases aggrecan gene expression in the meniscus. Am J Sports Med 26: 460-466.

13. Finsterbush A, Friedman B (1975) Reversibility of joint changes produced by immobilization in rabbits. Clin Orthop Relat Res : 290-298.

14. Chang JK, Ho ML, Lin SY (1996) Effects of compressive loading on articular cartilage repair of knee joint in rats. Kaohsiung J Med Sci 12: 453-460.

15. Klein L, Heiple KG, Torzilli PA, Goldberg VM, Burstein AH (1989) Prevention of ligament and meniscus atrophy by active joint motion in a non-weight-bearing model. J Orthop Res 7: 80-85.

16. Wayne JS, McDowell CL, Shields KJ, Tuan RS (2005) In vivo response of polylactic acid-alginate scaffolds and bone marrow-derived cells for cartilage tissue engineering. Tissue Eng 11: 953-963.

17. Chang NJ, Lam CF, Lin CC, Chen WL, Li CF, et al. (2013) Transplantation of autologous endothelial progenitor cells in porous PLGA scaffolds create a microenvironment for the regeneration of hyaline cartilage in rabbits. Osteoarthritis Cartilage 21: 1613-1622.

18. Rego-Pérez I, Fernández-Moreno $M$, Deberg M, Pértega S, Fernández-López C, et al. (2011) Mitochondrial DNA haplogroups and serum levels of proteolytic enzymes in patients with osteoarthritis. Ann Rheum Dis 70: 646-652.

19. Howard JS, Mattacola CG, Romine SE, Lattermann C (2010) Continuous Passive Motion, Early Weight Bearing, and Active Motion following Knee Articular Cartilage Repair: Evidence for Clinical Practice. Cartilage 1: 276-286.

20. Trudel G, Himori K, Uhthoff HK (2005) Contrasting alterations of apposed and unapposed articular cartilage during joint contracture formation. Arch Phys Med Rehabil 86: 90-97.

21. Kiviranta I, Jurvelin J, Tammi M, Saamanen AM, Helminen HJ (1987) Weight bearing controls glycosaminoglycan concentration and articular cartilage thickness in the knee joints of young beagle dogs. Arthritis Rheum 30: 801-809.

22. Sood SC (1971) A study of the effects of experimental immobilisation on rabbit articular cartilage. J Anat 108: 497-507.

23. Behrens F, Kraft EL, Oegema TR Jr (1989) Biochemical changes in articular cartilage after joint immobilization by casting or external fixation. J Orthop Res 7: 335-343.

24. Haapala J, Arokoski JP, Hyttinen MM, Lammi M, Tammi M, et al. (1999) Remobilization does not fully restore immobilization induced articular cartilage atrophy. Clin Orthop Relat Res : 218-229.

25. Salter RB, Bell RS, Keeley FW (1981) The protective effect of continuous passive motion in living articular cartilage in acute septic arthritis: an experimental investigation in the rabbit. Clin Orthop Relat Res : 223-247. 